Fortschreibung der bisherigen Landespolitik dar, die Regierungsmannschaft der schwarz-gelben Koalition bleibt personell unverändert. Lediglich zwei Minister mussten ihre Ressorts tauschen. Nach seinem Sieg im parteiinternen Wettkampf mit dem hessischen Ministerpräsidenten Koch stellte Wulff bald nach der Landtagswahl durch seinen Rückzug vom Landesvorsitz der niedersächsischen CDU die Weichen für ein verstärktes Engagement in der Bundespolitik.

(10) Die Opposition im Niedersächsischen Landtag besteht nach dem Einzug der Partei „Die Linke“ nun aus drei Fraktionen, deren zukünftige Ausrichtung und mögliche Koalitionsbildung untereinander im Hinblick auf die nächsten Landtagswahlen noch ungeklärt sind. Vor allem die SPD steht vor der Frage, wie sie sich richtungspolitisch orientieren und personalpolitisch aufstellen will.

\title{
Die Wähler der Linkspartei in ihren Hochburgen: eine empirische Analyse zur niedersächsischen Landtagswahl 2008 in Oldenburg, Delmenhorst und Wilhelmshaven
}

\author{
Manuela Kulick und Holger Onken
}

Bei der niedersächsischen Landtagswahl am 27. Januar 2008 erreichte die Linkspartei 7,1 Prozent der Stimmen und überwand damit für viele überraschend deutlich die Fünfprozenthürde. Nach ihrem Einzug in mittlerweile vier Parlamente westdeutscher Bundesländer findet die öffentliche Diskussion vor allem im Hinblick auf eine gewandelte Parteienlandschaft und die daraus folgenden veränderten Koalitionsoptionen statt. Die Ursachen des Wahlerfolges der Linkspartei werden dagegen vergleichsweise wenig untersucht.

Neue Parteien steigen häufig auf, weil Bürger mit der Umsetzung veränderter sozioökonomischer Bedingungen in politische Entscheidungen unzufrieden sind ${ }^{1}$. Sie werden demnach von einer Protesthaltung gegenüber der bestehenden politischen Ordnung getragen. Damit sich eine neue Partei aber langfristig im Parteiensystem etablieren kann, bedarf es mehr $^{2}$ : Sie muss eine Programmatik entwickeln, die eine tief greifende gesellschaftliche Konfliktlinie (Cleavage) abbildet, und von bestimmten sozialen (Groß-)Gruppen als am ehesten geeignet angesehen werden, ihre Interessen zu vertreten. Die Lebensdauer einer neuen Partei hängt maßgeblich davon ab, ob sich auf dieser Grundlage eine verlässliche, langfristig mobilisierbare Wählerbasis für sie entwickelt oder ob es beim kurzfristigen Protestverhalten bleibt. Das gilt auch für die Linkspartei in den westdeutschen Bundesländern.

1 Vgl. Hiltrud Naßmacher, Auf- und Abstieg von Parteien, in: ZParl, 20. Jg. (1989), H. 3, S. 170.

2 Die Institutionalisierung von Parteien hängt des Weiteren von Bedingungen des politischen Systems und dem Verhalten politischer Eliten ab; vgl. Ulrich Eith, Zur Ausprägung des politischen Wettbewerbs in entwickelten Demokratien. Zwischen gesellschaftlichen Konfliktlinien und dem Handeln politischer Eliten, in: ders. / Gerd Mielke (Hrsg.), Gesellschaftliche Konflikte und Parteiensysteme, Wiesbaden 2001, S. 17 - 33. 
Einer kurzfristigen Protestwahl können affektive Komponenten oder auch rationale Motive zugrunde liegen ${ }^{3}$. Eine affektive Protestwahl ist auf Wut und Enttäuschung über die etablierten Parteien zurückzuführen. Bei einer rationalen Protestwahl wird eine als Protestpartei angesehene Gruppierung gewählt, die mit einer bestimmten Politik in Verbindung gebracht wird, weil die etablierten Parteien sich zu weit von dem entfernt haben, was die Wähler für wünschenswert halten ${ }^{4}$. Korrigieren die etablierten Parteien ihre Programmatik oder ihr Regierungshandeln in die von den Wählern gewünschte Richtung, ist auch ein Rückgang der rationalen Protesthaltung zu erwarten. Berücksichtigt werden muss bei dieser Betrachtungsweise, dass bisher überwiegend rechtsextreme Parteien mit kurzfristigem Protestwahlverhalten in Verbindung gebracht werden. Deren öffentliche Stigmatisierung ${ }^{5}$ ist in Deutschland weit ausgeprägter als bei der Linkspartei, die bereits in drei der neuen Bundesländer an der Regierung eines Landes beteiligt war beziehungsweise ist.

Die Wahlerfolge der Linkspartei in Westdeutschland können aber auch ein Anzeichen dafür sein, dass sich die Partei dort zumindest mittelfristig etabliert. Die aus der Fusion der ostdeutschen PDS mit der westdeutschen WASG hervorgegangene „Die Linke“ könnte durch die WASG die im Westen für einen Wahlerfolg benötigte Legitimation erlangt haben, die der PDS seit der Wiedervereinigung gefehlt hatte ${ }^{6}$. Wenn es zutrifft, dass die Partei von bestimmten sozialen Gruppen als inzwischen legitime Vertreterin gemeinsamer Interessen angesehen wird, ist die Wahrscheinlichkeit, dass sich das Wahlverhalten für die Linke in Zukunft verfestigt, relativ hoch. Eine erste Exploration dieser Zusammenhänge soll hier für die Hochburgen der Linkspartei in Nordniedersachsen erfolgen, und zwar in Oldenburg, Delmenhorst und Wilhelmshaven ${ }^{7}$.

\section{Bestimmungsfaktoren für die Wahl der Linkspartei}

Wahlentscheidungen werden durch vielfältige Einflussfaktoren bestimmt. Häufig entsteht durch Diskussionen um die aktuelle Tagespolitik der Eindruck, kurzfristige, vermeintliche Erfolge oder Misserfolge einer bestimmten Partei wären ausschlaggebend für die Wahl. Stil und Inhalt solcher Diskussionen können für verschiedene Parteien durchaus unterschiedliche Mobilisierungseffekte haben. Bekanntermaßen geschehen Wahrnehmung und Beurteilung von Sachfragen und Kandidaten häufig vor dem Hintergrund einer langfristigen poli-

3 Vgl. Kai Arzheimer, Politikverdrossenheit: Bedeutung, Verwendung und empirische Relevanz eines politikwissenschaftlichen Begriffs, Wiesbaden 2001.

4 Franz Urban Pappi, Die Republikaner im Parteiensystem der Bundesrepublik. Protesterscheinung oder politische Alternative?, in: APuZ, B 21 (1990), S. 38 f.

5 Vgl. Frank Decker, Perspektiven des Rechtspopulismus in Deutschland am Beispiel der „SchillPartei“, in: APuZ, B 21 (2002), S. 24.

6 Vgl. Franz Walter, Eliten oder Unterschichten? Die Wähler der Linken, in: Tim Spier / Felix Butzlaff / Matthias Micus / Franz Walter (Hrsg.), Die Linkspartei. Zeitgemäße Idee oder Bündnis ohne Zukunft?, Wiesbaden 2007, S. $330 \mathrm{ff}$.

7 Bei der empirischen Auswertung wird auf Daten zurückgegriffen, die von der Arbeitsgruppe Empirische Demokratieforschung der Carl von Ossietzky Universität Oldenburg in Form einer direkten Nachwahlbefragung erhoben wurden. Insgesamt wurden 2241 Wähler befragt, die in Oldenburg, Wilhelmshaven und Delmenhorst in nach Repräsentativitätskriterien ausgewählten Wahllokalen zur Wahl gegangen waren. Der Fragebogen umfasste 30 Fragen und wurde anonym ausgefüllt. 
tischen Sozialisation der Wähler innerhalb bestimmter sozialer Gruppen ${ }^{8}$. Insgesamt wird der Zugehörigkeit zu einer sozialen Gruppe allerdings eine abnehmende Bedeutung für die Wahlentscheidung zugesprochen ${ }^{9}$, wobei dies vor allem für die ehemals stabilen sozialen Großgruppen gilt; an ihre Stelle ist eine Vielzahl kleinerer Gruppierungen getreten ${ }^{10}$.

\subsection{Sozialstrukturelle Bestimmungsfaktoren}

Durch den Erfolg der Linkspartei bei der Bundestagswahl 2005 und ihren anschließenden Einzug in vier westdeutsche Landesparlamente hat sich verglichen mit der PDS bei früheren Wahlen nicht nur die Anzahl ihrer Wähler deutlich vergrößert. Vielmehr hat sich auch ihr sozialstruktureller Hintergrund verschoben ${ }^{11}$.

Ein deutlich erkennbares Muster ergibt sich zunächst aus einem Stadt-Land-Vergleich bei der niedersächsischen Landtagswahl. Erwartungsgemäß schnitt die Linke in größeren Städten überwiegend besser ab als auf dem Land. In den Wahlkreisen der Städte mit mehr als 75.000 Einwohnern erreichte sie im Durchschnitt 9,2 Prozent; in den übrigen, überwiegend ländlich geprägten Wahlkreisen 6,6 Prozent. Jedoch konnte die Partei auch in ländlichen Wahlkreisen insbesondere in Nord- und Ostniedersachsen überdurchschnittliche Ergebnisse erzielen, vor allem in solchen mit relativ hoher Arbeitslosigkeit. Besonders in einigen Gegenden Ostfrieslands, der Umgebung von Cuxhaven und in mehreren direkt an Bremen grenzenden Wahlkreisen war die Partei erfolgreich. Lediglich in Wahlkreisen mit überwiegend katholischer Bevölkerung im Westen Niedersachsens blieb die Linkspartei unter fünf Prozent, hier erreichte sie im Durchschnitt nur 4,2 Prozent ${ }^{12}$.

Ein deutlicher Zusammenhang zwischen der Konfessionszugehörigkeit und der Wahlentscheidung zeigt sich auch bei den Linkswählern in den drei ausgewählten nordniedersächsischen Hochburgen. Auffällig ist das weit überdurchschnittliche Ergebnis unter Konfessionslosen, die in der Gesamtstichprobe über ein Viertel ausmachen. Unter diesen erreicht die Linkspartei 21 Prozent $^{13}$. Mehr als jeder zweite Befragte (54,3 Prozent) gehört der evangelischen Konfession an; innerhalb dieser Gruppe konnte die Linkspartei 11,2 Prozent für sich mobilisieren, womit sie geringfügig unter ihrem Durchschnitt liegt. Unter Katholiken, 15,5 Prozent in der Gesamtstichprobe, erreichte sie noch 8,2 Prozent. Im Vergleich zum schwachen Abschneiden im überwiegend katholisch und ländlich geprägten Südwesten Niedersachsens kommt die Linkspartei bei den in ihren städtischen Hochburgen wohnenden Katholiken somit auf ein deutlich besseres Ergebnis.

8 Vgl. Thomas von Winter, Politische Orientierungen und Sozialstruktur, Frankfurt am Main 1987, S. $139 \mathrm{ff}$.

9 Vgl. Wolfgang Jagodzinski / Markus Quandt, Wahlverhalten und Religion im Lichte der Individualisierungsthese: Anmerkungen zu dem Beitrag von Schnell und Kohler, in: Kölner Zeitschrift für Soziologie und Sozialpsychologie, 49. Jg. (1997), S. $761-782$.

10 Vgl. Stefan Hradil / Jürger Schiener, Soziale Ungleichheit in Deutschland, Wiesbaden 2005.

11 Vgl. Oskar Niedermayer, Die Wählerschaft der Linkspartei.PDS: sozialstruktureller Wandel bei gleich bleibender politischer Orientierung, in: ZParl, 37. Jg. (2006), H. 3, S. $524 \mathrm{ff.}$

12 Niedersächsischer Landeswahlleiter, http://cdl.niedersachsen.de/blob/images/C44870944_L20. pdf (10. März 2008); eigene Berechnungen.

13 Das gewichtete Stichprobenergebnis der Linkspartei liegt mit 13,5 Prozent über dem durchschnittlichen Ergebnis in den drei Städten, in denen die Daten erhoben wurden (10,4 Prozent). 
Bei den Berufsgruppen schneidet die Linkspartei erwartungsgemäß unter Arbeitern mit mehr als 25 Prozent der Stimmen überdurchschnittlich gut ab. In der Gruppe der Arbeitslosen gab ihr sogar jeder Dritte seine Stimme. Bei Angestellten erzielte die Linkspartei ein Ergebnis, das mit 14,4 Prozent etwas über ihrem Durchschnitt lag.

Aus der Zugehörigkeit zu einer Berufsgruppe und den Einkommensverhältnissen lässt sich die Gruppe der Modernisierungsverlierer konstruieren ${ }^{14}$. Dieser können 22 Prozent aller Befragten zugerechnet werden. Modernisierungsverlierer votieren deutlich überproportional für die Linkspartei; 37,8 Prozent ihrer Wähler lassen sich dieser Gruppe zuordnen. Diese Wählerschaft scheint also eine Seite der nach wie vor für das deutsche Parteiensystem strukturvermittelnden sozioökonomischen Konfliktlinie ${ }^{15} \mathrm{zu}$ repräsentieren. Sie bevorzugt einen von der Linkspartei programmatisch vertretenen staatlichen Interventionismus gegenüber einem Marktliberalismus, der am stärksten von der FDP verfochten wird. Folgerichtig sind Modernisierungsverlierer innerhalb der FDP-Wählerschaft mit zwölf Prozent am deutlichsten unterrepräsentiert.

Das Ergebnis der Linkspartei unter den Modernisierungsverlierern deckt sich mit ihren Resultaten in den niedrigen Einkommensklassen (bei ausschließlicher Betrachtung des Haushaltsnettoeinkommens): In den Wählergruppen, die in Haushalten mit weniger als 1000 Euro und solchen mit einem Haushaltsnettoeinkommen zwischen 1000 bis unter 2000 Euro leben, ist die Linke mit Wahlergebnissen von 19,0 beziehungsweise 16,4 Prozent überrepräsentiert. Mit 12,4 Prozent in der mittleren Einkommensklasse (von 2000 bis unter 3000 Euro) schneidet die Partei immer noch relativ gut ab. Lediglich in der Klasse über 3000 Euro liegt sie mit 7,4 Prozent deutlich unter ihrem Durchschnitt.

Betrachtet man die Wähler der Linkspartei nach Bildungsabschlüssen, gibt es kaum Abweichungen von der Verteilung unter allen Befragten. 21,3 Prozent der Linksparteiwähler gaben an, einen Haupt- oder Volksschulabschluss zu haben, in der Gesamtstichprobe waren es 21,4 Prozent. Auch bei den Befragten mit Realschulabschluss gab es, ebenso wie bei solchen mit Abitur oder Fachabitur, kaum Unterschiede zwischen der Zusammensetzung der Stichprobe und der Linksparteiwählerschaft: Von ihnen hatten 26,6 Prozent einen Realschulabschluss, im Verhältnis zu 27,7 Prozent aller Befragten. Jeder fünfte Wähler der Linkspartei verfügt über das Abitur oder Fachabitur, was in etwa dem Anteil dieser Gruppe in der Gesamtstichprobe (21,3 Prozent) entspricht. Lediglich die Hochschul- und Fachhochschulabsolventen sind in der Wählerschaft der Linkspartei mit 31,2 Prozent im Verhältnis zu ihrem Anteil an allen Befragten (28,8 Prozent) geringfügig überrepräsentiert.

Hinsichtlich der Altersstruktur der Linkspartei fällt auf, dass sie in den beiden jüngeren Klassen (bis unter 35 Jahre) mit 12,5 Prozent leicht und in der ältesten Klasse (über 65-Jährige) mit 7,8 Prozent stark unterrepräsentiert ist. Ihr bestes Resultat erzielt sie mit 17,2 Prozent in der Gruppe der 35- bis unter 50-Jährigen. Bei den 50- bis unter 65-Jährigen

$14 \mathrm{Zu}$ den Modernisierungsverlierern wurden in dem von Oskar Niedermayer, Bettina Westle und Steffen Kühnel geleiteten DFG-Projekt „Bürger und Parteien“ (2005) Arbeitslose, Arbeiter, einfache Angestellte und Beamte im einfachen Dienst gezählt, deren Haushaltsnettoeinkommen unter 1500 Euro liegt. Vgl. Oskar Niedermayer, a.a.O. (Fn. 11); hier wird diese Gruppe mit bis unter 2000 Euro Haushaltsnettoeinkommen etwas weiter gefasst, da die Daten entsprechend erhoben wurden.

15 Vgl. Franz Urban Pappi, Die politisierte Sozialstruktur heute: Historische Reminiszenz oder aktuelles Erklärungspotential?, in: Frank Brettschneider / Jan van Deth / Edeltraud Roller (Hrsg.), Das Ende der politisierten Sozialstruktur?, Opladen 2002, S. $41 \mathrm{ff}$. 
schneidet sie mit 15 Prozent leicht überdurchschnittlich ab. Männer sind mit 53,3 Prozent in der Linksparteiwählerschaft geringfügig überrepräsentiert. Diese Befunde aus den drei nordniedersächsischen Städten decken sich weitgehend mit Ergebnissen, die auf Wahltagsbefragungen in ganz Niedersachsen beruhen ${ }^{16}$.

\subsection{Einstellungs- und themenbezogene Bestimmungsfaktoren}

Die Position in der Sozialstruktur bildet eine wichtige Grundlage für die individuelle politische Einstellung und die Bewertung bestimmter politischer Sachfragen sowie die damit in Verbindung gebrachten Parteien und ihres Personals ${ }^{17}$. Auch für die sozialstrukturelle Position, der sich die Wähler selbst zuordnen, ist ihre tatsächliche Position in der Sozialstruktur wichtig ${ }^{18}$. Nach der im Herbst 2006 auf Grundlage einer Studie der Friedrich-Ebert-Stiftung geführten Diskussion um das „abgehängte Prekariat“ kam die Frage auf, ob diese Gruppe, die etwa vier Prozent der Bevölkerung in Westdeutschland ausmachen soll, politisch bindungsfähig sei oder nicht. Seitdem wird die Linkspartei in der öffentlichen Diskussion häufig in Verbindung mit der so genannten Unterschicht gebracht ${ }^{19}$. Diese Annahme lässt sich durch die Stichprobe in Nordniedersachsen empirisch belegen.

\begin{tabular}{|l|c|c|c|c|c|c|}
\hline Tabelle 1: Subjektive Schichtzugehörigkeit der Parteiwählerschaften in drei Städten \\
Nordniedersachsens bei der Landtagswahl 2008 (Angaben in Prozent) \\
\hline & CDU & SPD & FDP & Grüne & Linke & Alle \\
\hline Unterschicht / untere Mittelschicht & 17,7 & 27,9 & 14,8 & 23,3 & $\mathbf{4 6 , 7}$ & 25,5 \\
\hline Mittelschicht & $\mathbf{6 0 , 3}$ & $\mathbf{5 8 , 2}$ & $\mathbf{5 9 , 9}$ & $\mathbf{6 0 , 4}$ & 44,9 & $\mathbf{5 7 , 6}$ \\
\hline Obere Mittelschicht / Oberschicht & 22,0 & 13,9 & 25,4 & 16,3 & 8,4 & 17,0 \\
\hline $\begin{array}{l}\text { N = 1726. } \\
\text { Quelle: Eigene Erhebung. }\end{array}$
\end{tabular}

Eine deutliche Mehrheit der hier Befragten ordnet sich der Mittelschicht zu (vgl. Tabelle 1). Die Wähler der Linkspartei weichen insgesamt am deutlichsten von der Verteilung unter allen Befragten ab. Sie gruppieren sich erheblich häufiger in die unteren gesellschaftlichen Schichten ein als die Wähler aller anderen Parteien. Innerhalb der Gruppe, die sich als Angehörige der Unterschicht bezeichnen (etwa fünf Prozent von allen Befragten), erreichte die Linkspartei über 40 Prozent und ist damit in dieser Gruppe die stärkste Partei. Wähler, die sich zur unteren Mittelschicht zählen (20,5 Prozent aller Befragten), stimmten mit 20 Prozent immer noch deutlich überproportional für die Linke.

Auch bei der Zufriedenheit mit der Landes- beziehungsweise Bundesregierung weichen die Wähler der Linkspartei deutlich von denen der anderen Parteien ab: Sie sind mit Abstand am unzufriedensten. Auf einer Skala von 1 (gar nicht zufrieden) bis 10 (sehr zufrie-

16 Vgl. den Beitrag von RalfTils und Thomas Saretzki in diesem Heft der ZParl.

17 Vgl. Harald Schoen, Gründe für wechselndes Wahlverhalten: Helfen neue Instrumente Licht in das Dunkel zu bringen?, in: PVS, 41. Jg. (2000), S. 677 - 706.

18 Dabei kann eine soziologische Kategorisierung durchaus von der Selbsteinschätzung der Wähler abweichen.

19 Vgl. zum Beispiel: Die Zeit vom 31. Januar 2008. 
den) erreichte die Zufriedenheit mit der Landesregierung bei den Linkswählern 2,7; unter allen Befragten betrug der Wert 4,9. Die Unzufriedenheit mit der Großen Koalition im Bund war unter den Wählern der Linken mit 2,3, aber auch unter allen Befragten mit 4,4 noch ausgeprägter.

Ein Zusammenhang ergibt sich auch zwischen der Erwartung, wie sich die eigene Lebenssituation in den nächsten fünf Jahren entwickeln wird, und der Wahlentscheidung für die Linkspartei (vgl. Tabelle 2). Die Wähler der Linkspartei sind die einzigen, die eine Verschlechterung ihrer Lebensbedingungen erwarten. Diese Einschätzung zusammen mit ihrer Selbstverortung innerhalb der gesellschaftlichen Schichtung wirken sich auch auf die $\mathrm{Zu}-$ friedenheit mit der Demokratie aus. Die Linksparteiwähler, die sich überproportional den unteren Bereichen der Gesellschaft zuordnen und überwiegend eine Verschlechterung der eigenen Lebenssituation erwarten, drücken als einzige Wählergruppe überwiegend Unzufriedenheit mit der Demokratie aus (vgl. Tabelle 3).

\begin{tabular}{|l|c|c|c|}
\hline Tabelle 2: Beurteilung der Entwicklung der Lebenssituation in den nächsten fünf Jahren in drei \\
Städten Nordniedersachsens bei der Landtagswahl 2008
\end{tabular}

\begin{tabular}{|c|c|c|c|}
\hline \multicolumn{4}{|c|}{$\begin{array}{l}\text { Tabelle 3: Zufriedenheit mit der Demokratie in Deutschland in drei Städten Nordniedersachsens } \\
\text { bei der Landtagswahl } 2008\end{array}$} \\
\hline & Mittelwert & $\mathrm{N}$ & $\begin{array}{c}\text { Standard- } \\
\text { abweichung }\end{array}$ \\
\hline SPD & 5,9 & 605 & 2,45 \\
\hline $\mathrm{CDU}$ & 6,7 & 553 & 2,25 \\
\hline B90/Grüne & 5,8 & 257 & 2,31 \\
\hline FDP & 6,4 & 146 & 2,26 \\
\hline Die Linke & 4,1 & 249 & 2,27 \\
\hline Alle & 5,9 & 1810 & 2,47 \\
\hline \multicolumn{4}{|c|}{$\begin{array}{l}\text { Wortlaut der Frage: Wie zufrieden sind Sie mit der Demokratie in Deutschland? Die Skala reicht von } 1 \\
\text { (gar nicht zufrieden) bis } 10 \text { (sehr zufrieden). } \\
\mathrm{N}=1810 \text {. } \\
\text { Quelle: Eigene Erhebung. }\end{array}$} \\
\hline
\end{tabular}


Zwar lässt ein hohes Maß an Unzufriedenheit mit der Demokratie nicht notwendigerweise Rückschlüsse darauf zu, dass die Demokratie als Ganzes abgelehnt wird. Eine gewisse Protesthaltung der Linksparteiwähler kann daraus aber abgeleitet werden. Wie diese Haltung genau einzuschätzen ist, bestimmt maßgeblich, wie langfristig die Erfolge der von Protest getragenen Parteien sind. Entscheidend für eine mittel- oder langfristige Etablierung einer neuen Partei ist, wie oben skizziert, ob sich durch sozialen und ökonomischen Wandel eine neue gesellschaftliche Konfliktlinie, die für das Parteiensystem dauerhaft eine strukturierende Wirkung entfaltet, entwickelt ${ }^{20}$.

Die Bedeutung, die die Wähler einzelnen Themen zusprechen, hängt ebenfalls, zumindest indirekt, davon ab, wie sie sich in der Sozialstruktur einordnen ${ }^{21}$. Die Befragten hatten Gelegenheit, unter 18 Themen bis zu drei auszuwählen, die für ihre Wahlentscheidung besonders wichtig waren (vgl. Tabelle 4). Nur die Bildung, als überwiegend landespolitisches Thema, ist bei den Wählern aller Parteien unter den drei am häufigsten genannten. Unter den Anhängern der Linkspartei hielt knapp jeder Zweite Bildung für sehr wichtig; 43,1 Prozent gaben Mindestlohn an. Bereits bei der Bundestagswahl 2005 waren für 61 Prozent der Linksparteiwähler soziale Gerechtigkeit und die damit in Verbindung gebrachten Themen Bildung und Mindestlohn von großer Bedeutung ${ }^{22}$. Etwa ein Viertel der Wähler der Linkspartei zählen Klimaschutz, Energiepolitik und Gesundheitspolitik zu den wichtigsten Fragen für ihre Wahlentscheidung. Unter jenen, die Klimaschutz und Energiepolitik nannten, sind überproportional viele zu finden, die bei der Landtagswahl 2003 noch für die Grünen votiert hatten.

\begin{tabular}{|c|c|c|c|c|c|}
\hline \multicolumn{6}{|c|}{$\begin{array}{l}\text { Tabelle 4: Am häufgsten genannte, für die Wablentscheidung wichtigste Themen innerhalb der } \\
\text { Parteianhängerschaften in drei Städten Nordniedersachsens bei der Landtagswabl } 2008 \\
\text { (Angaben in Prozent) }\end{array}$} \\
\hline & $\mathrm{CDU}$ & SPD & FDP & Grüne & Linke \\
\hline Bildung & 44,5 & 51,0 & 49,2 & 55,1 & 47,2 \\
\hline Klimaschutz & 23,2 & 34,6 & 27,8 & 70,1 & 27,5 \\
\hline Mindestlohn & 11,8 & 45,4 & 9,2 & 16,9 & 43,1 \\
\hline Energiepolitik & 20,9 & 21,1 & 17,9 & 39,3 & 22,9 \\
\hline Gesundheitspolitik & 23,5 & 24,2 & 31,4 & 10,1 & 24,5 \\
\hline Finanzpolitik & 35,3 & 13,3 & 36,0 & 2,8 & 16,6 \\
\hline Kinderbetreuung & 22,2 & 17,8 & 13,2 & 17,6 & 16,7 \\
\hline \multicolumn{6}{|c|}{$\begin{array}{l}\text { Mehrfachantworten waren möglich. } \\
\mathrm{N}=1359 . \\
\text { Quelle: Eigene Erhebung. }\end{array}$} \\
\hline
\end{tabular}

20 Vgl. Klaus von Beyme, Parteien in westlichen Demokratien, München 1982, S. 249 ff.

21 Vgl. Geoffrey C. Layman / Thomas M. Carsey, Party Polarization and Party Structuring of Policy Attitudes: A Comparison of Three NES Panel Studies, in: Political Behavior, 24. Jg. (2002), S. $199-236$.

22 Vgl. Gero Nengebauer / Richard Stöss, Die Partei die Linke. Nach der Gründung in des Kaisers neuen Kleidern? Eine politische Bedarfsgemeinschaft als neue Partei im deutschen Parteiensystem, in: Oskar Niedermayer (Hrsg.), Die Parteien nach der Bundestagswahl 2005, Wiesbaden 2008, S. 162 f. 
Die Wertvorstellungen der Wähler sind ein weiterer Bestimmungsgrund für die Wahlentscheidung und die Bindung an eine Partei, die mit diesen Werten in Verbindung gebracht wird. Wertvorstellungen wirken strukturierend auf die Beurteilung einzelner Themenbereiche und der programmatischen Aussagen der Parteien zu bestimmten Sachfragen ${ }^{23}$. Der Zusammenhang zwischen als persönlich besonders wichtig empfundenen Werten und der Wahlentscheidung für eine bestimmte Partei wird aus Tabelle 5 ersichtlich. Soziale Gerechtigkeit wird von mehr als 90 Prozent der befragten Linksparteiwähler in den drei ausgewählten Städten angegeben; eine so hohe Konzentration ist bei keiner anderen Partei zu finden. Dass die Wählerschaft der Linkspartei bei den in der Verantwortung stehenden Parteien Defizite in diesem Bereich sieht, wird durch das hohe Maß ihrer Unzufriedenheit mit der Landes- und insbesondere der Bundesregierung deutlich. Soziale Gerechtigkeit ist demnach das Band, das die Wählerschaft der Linkspartei eint; „Die Linke“ wird von ihren Wählern offenbar mit sozialer Gerechtigkeit und den damit in Verbindung gebrachten Themen identifiziert.

\begin{tabular}{|c|c|c|c|c|c|}
\hline \multicolumn{6}{|c|}{$\begin{array}{l}\text { Tabelle 5: Persönlich als besonders wichtig empfundene Werte unter den Parteianhängerschaften } \\
\text { drei Städten Nordniedersachsens bei der Landtagswahl } 2008 \text { (Angaben in Prozent) }\end{array}$} \\
\hline & $\mathrm{CDU}$ & SPD & FDP & Grüne & Linke \\
\hline Soziale Gerechtigkeit & 50,8 & 79,7 & 50,2 & 77,4 & 90,7 \\
\hline Persönliche Freiheit & 39,5 & 35,3 & 49,0 & 25,4 & 39,7 \\
\hline Familie & 39,8 & 33,9 & 29,0 & 21,4 & 24,0 \\
\hline Innere Sicherheit & 45,2 & 22,1 & 41,6 & 6,0 & 13,6 \\
\hline Umweltschutz & 14,0 & 20,0 & 16,1 & 65,3 & 23,4 \\
\hline \multicolumn{6}{|c|}{$\begin{array}{l}\text { Die Wähler sollten unter den angeführten Werten maximal zwei angeben. } \\
\mathrm{N}=1687 . \\
\text { Quelle: Eigene Erhebung. }\end{array}$} \\
\hline
\end{tabular}

\section{Binnenstruktur der Linksparteiwählerschaft}

Bisher kann festgehalten werden, dass sich die Wählerschaft der Linkspartei von den Wählerschaften der anderen Parteien sowohl im Hinblick auf sozialstrukturelle Eigenschaften als auch auf einstellungs- und themenbezogene Präferenzen unterscheidet. Insgesamt kommt das Profil der SPD-Wähler dem der Linksparteiwähler deutlich näher als das der CDU-, FDP- und Grünen-Wähler. Um Anhaltspunkte dafür zu erhalten, in welchem Maße die Linke in ihren Hochburgen bereits über ein längerfristig mobilisierbares Wählerpotenzial verfügt, wird nachfolgend ein Schwerpunkt auf die Unterschiede innerhalb der Linksparteiwählerschaft gelegt. Im Mittelpunkt stehen Indikatoren, die auf feste Wählerbindungen an die Partei schließen lassen beziehungsweise solche, die eher kurzfristiges Protestwahlverhalten nahe legen.

23 Wolfgang Jagodzinski, Sozialstruktur, Wertorientierungen und Parteibindungen: Zur Problematik eines Sozialisationsmodells, in: Zeitschrift für Soziologie, 10. Jg. (1981), S. 170 - 191, S. 171 f. 


\subsection{Parteiidentifikation}

Wähler, die sich mit einer Partei identifizieren, werden als überzeugte Anhänger dieser Partei angesehen ${ }^{24}$. Als Äquivalent zu der üblicherweise verwendeten, sehr umfangreichen Fragestellung zur Ermittlung der Parteiidentifikation sollten die Wähler in der vorliegenden Umfrage angeben, ob sie sich einer Partei verbunden fühlen. Diejenigen, die dies bejahten und hierbei die Linke nannten, werden als überzeugte Anhänger angesehen; diese Gruppe macht 21,4 Prozent der befragten Linksparteiwähler aus und ist damit deutlich kleiner als bei den anderen Parteien: SPD 52, CDU 41, Grüne 48 und FDP 30 Prozent. Daraus könnte man schlussfolgern, dass ein hoher Anteil an ungebundenen Protestwählern unter den Linksparteiwählern zu finden ist. Da aber in der Wahlforschung davon ausgegangen wird, dass die Identifikation mit einer Partei in sehr langfristigen Sozialisationsprozessen erworben wird ${ }^{25}$, kann der Anteil der parteiverbundenen Wähler der erst seit kurzem existierenden Linkspartei mit über 20 Prozent als relativ hoch bewertet werden. In dieser Gruppe ist auch der Anteil der Wähler, der angab, bei der Landtagswahl 2003 bereits die PDS gewählt zu haben, mit fast 60 Prozent am höchsten, was deutlich dafür spricht, dass einer Bindung an eine bestimmte Partei häufig ein langfristiger Prozess vorausgeht.

\subsection{Wahlverhalten in der Vergangenheit}

Aus einer wiederholten Stimmabgabe für eine Partei lässt sich nicht notwendigerweise Verbundenheit beziehungsweise Identifikation mit dieser ableiten, wohl aber deutet dies auf ein längerfristig mobilisierbares Wählerpotenzial hin. Zudem führt eine wiederholte Stimmabgabe für dieselbe Partei häufig zu wachsender Loyalität ${ }^{26}$. Ein Teil der befragten Wählerschaft der Linkspartei gab an, bereits bei der Bundestagswahl 2005 für sie gestimmt zu haben. Zudem wurde nach der Wahlabsicht bei der nächsten Bundestagswahl ${ }^{27}$ gefragt. Die Linksparteiwähler, die bekundeten, 2005 für die Linke gestimmt zu haben und sie bei der nächsten Bundestagswahl wählen zu wollen, können als ziemlich überzeugte Anhängerschaft der Partei angesehen werden, obwohl sie explizit keine Verbundenheit mit dieser angaben. Diese Gruppe macht 15 Prozent der Linksparteiwählerschaft aus.

\subsection{Rationale Protesthaltung}

10,7 Prozent der befragten Linksparteiwähler fühlten sich der SPD verbunden. Einen solch hohen Wert einer vom Wahlverhalten abweichenden Parteiverbundenheit gibt es bei keiner

24 Vgl. Wilhelm Bürklin / Markus Klein, Wahlen und Wählerverhalten, Opladen 1998, S. 66 f.

25 Vgl. Kai Arzheimer / Jürgen W. Falter, Wahlen, in: Eckhard Jesse / Roland Sturm (Hrsg.), Demokratien des 21. Jahrhunderts, Opladen 2003, S. 299 f.

26 Vgl. Kenneth J. Meier, Party Identification and Vote Choice: The Causal Relationship, in: Western Political Quarterly, 28. Jg. (1975), S. 496 - 505.

27 Bei Bundestagswahlen ist Protestwahlverhalten weit schwächer ausgeprägt als bei Landtagswahlen (vgl. Wolfgang Rudzio, Das politische System der Bundesrepublik Deutschland, 6. Auflage, Opladen 2003, S. 228); insofern spricht die Absicht, bei der nächsten Bundestagswahl für die Linke $\mathrm{zu}$ votieren, gegen eine kurzfristige Protestwahl. 
anderen Partei. Diese Wähler wollen offenbar mit ihrer Stimmabgabe eine linker ausgerichtete Politik der SPD erzwingen. Sie haben demnach eine eindeutige programmatische Präferenz; sollte die SPD sich auf lange Sicht für einen relativ angebotsorientierten wirtschaftspolitischen Kurs entscheiden, könnte dieses Wählersegment auch längerfristig seine politische Heimat in der Linkspartei sehen. Die ihm Angehörigen engagieren sich deutlich häufiger ehrenamtlich in gesellschaftlichen Organisationen wie Gewerkschaften ${ }^{28}$, karitativen Einrichtungen, Kirchen usw. als die übrigen Wähler der Linkspartei. In dieser Gruppe ist demnach eine politisch besonders aktive Klientel enthalten, die häufig aus dem Umfeld der Kritiker der „Agenda 2010-Politik“ stammt ${ }^{29}$.

12,8 Prozent der Linksparteiwähler können als affektive Protestwähler eingeordnet werden. Sie zeichnen sich durch häufige Wechsel ihrer Parteipräferenzen aus, die keinem erkennbaren Muster folgen. Darunter sind viele, die bei der letzten Landtagswahl 2003 und / oder der Bundestagswahl 2005 für die CDU stimmten, sowie ehemalige Nichtwähler.

Gut 40 Prozent der befragten Linksparteiwähler in den drei nordniedersächsischen Hochburgen lassen sich weder als Protestwähler noch als überzeugte Anhänger eindeutig charakterisieren. Bei ihnen handelt es sich um ehemalige Wähler der SPD oder der Grünen, die sich im Gegensatz zu den rationalen Protestwählern keiner Partei verbunden fühlen. Eine nur einmalige Stimmabgabe für die Linkspartei durch die dieser Gruppe zugerechneten Wähler ist eher unwahrscheinlich, da über 90 Prozent von ihnen angaben, auch bei der nächsten Bundestagswahl für die Linkspartei stimmen zu wollen ${ }^{30}$. Diese Wähler scheinen also kein lagerübergreifendes Wahlverhalten in Erwägung zu ziehen und können daher als potenzielle Stammwähler der Linkspartei angesehen werden. Von den überzeugten Anhängern unterscheiden sie sich dadurch, dass sie bei der Bundestagswahl 2005 nicht für die Linkspartei votierten. Die dieser Gruppe zugehörigen Wähler scheinen sich aus Enttäuschung über die von der rot-grünen Regierung eingeleiteten Reformen der Agenda 2010 und der Hartz IV-Gesetzgebung von den ehemaligen Regierungsparteien im Bund abgewendet zu haben. Vor allem enttäuschte linke Sozialdemokraten verließen die SPD in den alten Bundesländern und gründeten 2004 die WASG. Aufgrund des 2007 erfolgten Zusammenschlusses der WASG mit der PDS unter dem unbelasteten Namen „Die Linke“ sank die Hemmschwelle für bisherige SPD- und Grünen-Wähler, der Partei ihre Stimme zu geben $^{31}$. Auch die Zusammenarbeit mit führenden Gewerkschaftsvertretern dürfte dazu beigetragen haben, denn unter den potenziellen Stammwählern finden sich viele den Gewerkschaften nahestehende Personen. Ob die Linkspartei diese Wähler dauerhaft gewinnen und sich als dauerhafte Alternative innerhalb des linken Lagers etablieren kann, hängt maßgeblich davon ab, wie sich ihr personelles und programmatisches Angebot entwickelt ${ }^{32}$.

28 Vgl. den Beitrag von Ralf Tils und Thomas Saretzki in diesem Heft der ZParl.

29 Vgl. Harald Schoen / Jürgen W. Falter, Die Linkspartei und ihre Wähler, in: APuZ, B 51/52 (2005), S. 33 - 40.

30 Das tatsächlich mobilisierbare Wählerpotenzial der Linkspartei in der Gesamtbevölkerung könnte durchaus höher liegen. Vgl. Renate Köcher, Die Sogwirkung der Linkspartei, in: FAZ vom 20. Februar 2008.

31 Vgl. Udo Baron / Manfred Wilke, Operation Vereinigung, in: Die politische Meinung, Nr. 439 (2006), S. 67, S. 73.

32 Vgl. Gero Neugebauer / Richard Stöss, a.a.O. (Fn. 22), S. 163 f. 


\subsection{Typologisierung der Linksparteiwähler}

Auf der Grundlage des Wahlverhaltens in der Vergangenheit und der Wahlabsichten in der Zukunft sowie nach den Angaben zur Parteiverbundenheit lässt sich die Wählerschaft der Linkspartei in den drei Städten, für die Daten vorliegen, in überzeugte Anhänger, potenzielle Stammwähler und Protestwähler kategorisieren (vgl. Abbildung 1). Auch wenn diese Wählertypologisierung nicht idealtypisch ${ }^{33}$, sondern allenfalls grob mit zum Teil erheblichen Überschneidungen der einzelnen Gruppen erfolgen kann, spricht für sie, dass es zwischen den Wählergruppen messbare Unterschiede gibt. Diese finden sich sowohl bei den sozialstrukturellen als auch bei den themen- und einstellungsbezogenen Merkmalen.

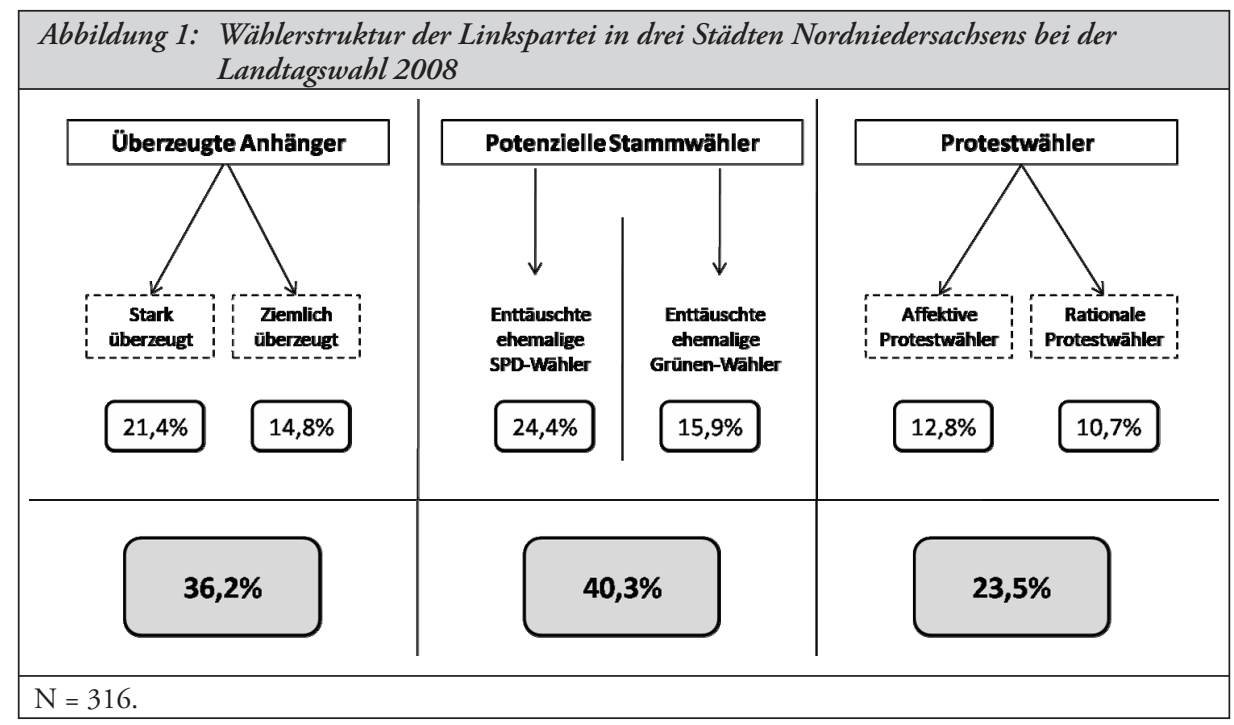

Auffällig ist die unterschiedliche Zusammensetzung der Gruppen im Hinblick auf die Bildungsabschlüsse: Unter den überzeugten Anhängern sind verhältnismäßig viele Wähler mit höheren Bildungsabschlüssen und eher selten solche mit Haupt- oder Realschulabschluss vertreten. Bei den Protestwählern finden sich überproportional viele Wähler mit formal niedrigeren Bildungsabschlüssen. Die Gruppe der potenziellen Stammwähler weist hinsichtlich der Schulbildung keine Besonderheiten auf.

Auch bei der Konfessionszugehörigkeit gibt es Unterschiede zwischen den Wählergruppen. Der Anteil der Konfessionslosen in der Wählerschaft der Linkspartei insgesamt ist mit 41,7 Prozent bereits deutlich höher als unter allen Befragten (25 Prozent). Von den überzeugten Anhängern ist sogar die Hälfte konfessionslos, Katholiken sind in dieser Gruppe dagegen kaum zu finden (drei Prozent). Es fallen zudem Unterschiede bei den Einkommensgruppen auf: Fast 42 Prozent der überzeugten Linksparteiwähler leben in Haushalten mit einem Nettoeinkommen von weniger als 1000 Euro; von denjenigen, die den Protest-

33 Zur Problematik der Wählertypologisierung vgl. Gerd Strohmeier, Moderne Wahlkämpfe - wie sie geplant, geführt und gewonnen werden, Baden-Baden 2002, S. $70 \mathrm{ff}$. 
wählern zugeordnet wurden, sind es dagegen nur 20 Prozent; innerhalb der Gruppe der potenziellen Stammwähler 31 Prozent. In dieser wie auch in den höheren Einkommensklassen entspricht die Verteilung der drei Teilgruppen fast genau jener der Linksparteiwählerschaft insgesamt. Die Kombination aus einem hohen Bildungsabschluss und niedrigem Einkommen erhöht demnach die Wahrscheinlichkeit, dass ein Wähler nach dem Schema in Abbildung $1 \mathrm{zu}$ den überzeugten Anhängern der Linkspartei gezählt wird.

Hinsichtlich der Altersstruktur ist festzustellen, dass unter den Protestwählern ältere Altersklassen stärker vertreten sind als jüngere. Die überzeugten Anhänger weisen eine gleichmäßige Altersverteilung auf. Unter denen, die zu den potenziellen Stammwählern gezählt werden, sind besonders viele 25- bis unter 35-Jährige zu finden, während Personen über 65 Jahre unterdurchschnittlich repräsentiert sind. Geschlechtsspezifische Unterschiede zwischen den Gruppen gibt es dagegen nicht.

Insgesamt gesehen entsprechen die potenziellen Stammwähler weitgehend der Verteilung innerhalb der Linksparteiwählerschaft in den drei untersuchten nordniedersächsischen Städten, während sich bei den überzeugten Anhängern und den Protestwählern hier Unterschiede zeigen. So sehen sich nur 8,4 Prozent der Linksparteiwähler als Angehörige der oberen Mittelschicht / Oberschicht, von den überzeugten Anhängern ordnen sich aber 11,5 Prozent dieser Schicht zu. Dieser Befund deckt sich damit, dass in dieser Gruppe überproportional viele Personen mit hohen Bildungsabschlüssen anzutreffen sind, widerspricht aber dem Umstand, dass viele dieser Wähler in Haushalten mit niedrigem Nettoeinkommen leben. Protestwähler ordnen sich dagegen überproportional häufig der Mittelschicht zu: 53 Prozent gegenüber knapp 45 Prozent von allen Linksparteiwählern. Dies kann als Indiz dafür gedeutet werden, dass verbreitete Ängste vor sozialem Abstieg zu Protestwahlverhalten führen können.

Auch in ihren allgemeinen politischen Einstellungen erweist sich die Wählerschaft der Linkspartei als eine durchaus heterogene Gruppe. Ein Großteil ihrer Wähler erwartet eine negative Entwicklung der eigenen Lebenssituation. Dabei sehen die Protestwähler dies etwas pessimistischer (Mittelwert $=3,63$ ) als die überzeugten Anhänger der Linkspartei $(3,34)^{34}$. Auf die Frage nach der Zufriedenheit mit der Demokratie in Deutschland gaben die Wähler der Linkspartei dieser schlechtere Werte als diejenigen der anderen Parteien. Hier sind insbesondere die überzeugten Anhänger der Linkspartei mit einem Mittelwert von 3,935 deutlich unzufriedener als die Protestwähler, die mit 5,0 über dem allgemeinen Durchschnitt der Linkspartei liegen (4,3). Den Durchschnittswert erreichen die potenziellen Stammwähler. Auch bei Fragen nach der Zufriedenheit mit der amtierenden Landesregierung und der Großen Koalition im Bund zeigten sich die Protestwähler stets zufriedener als die überzeugten Anhänger oder die potenziellen Stammwähler ${ }^{36}$. Diese Wähler sind nicht prinzipiell mit der Demokratie unzufrieden, aber offenbar mit der politischen Ausrichtung der etablierten Parteien, insbesondere der SPD.

34 Auf einer Skala von 1 (positiv) bis 5 (negativ); Mittelwert der Linksparteiwähler insgesamt 3,43.

35 Die Skala reichte von 1 (sehr unzufrieden) bis 10 (sehr zufrieden).

36 So gaben die Protestwähler bei der Frage nach der Zufriedenheit mit der Landesregierung im Mittel 2,9 an; die überzeugten Anhänger dagegen 2,4. Bei der Frage nach der Zufriedenheit mit der Großen Koalition ist der Unterschied größer, mit 2,0 der überzeugten Anhänger zu 2,8 der Protestwähler. Im Ganzen betrachtet sind sowohl Protest- als auch überzeugte Wähler unzufrieden mit den Regierungen, lediglich die Stärke der Unzufriedenheit variiert. 
Auch im Hinblick auf die Bedeutung verschiedener Themen und Politikbereiche gibt es zwischen den Gruppen unterschiedlich häufig genannte Präferenzen. Während knapp jeder zweite überzeugte Anhänger der Linkspartei und Protestwähler Mindestlohn zu den drei wichtigsten Themen zählt, trifft dies nur für 39 Prozent der potenziellen Stammwähler $\mathrm{zu}^{37}$. Auch Bildung war diesen nicht ganz so wichtig (39 Prozent) wie den überzeugten Anhängern (53,7 Prozent) und den Protestwählern (50 Prozent). Die überzeugten Anhänger wie auch die Protestwähler konzentrieren sich stärker auf wenige Themen, während sich die dritte Gruppe auf unterschiedliche Bereiche gleichmäßiger verteilt. Bei der Frage, welche Werte den Wählern persönlich besonders wichtig sind, gab es nur bei der „persönlichen Freiheit" messbare Unterschiede zwischen den verschiedenen Wählergruppen der Linkspartei. Diese wurde von immerhin jedem zweiten überzeugten Anhänger genannt, während es in den beiden anderen Gruppen nur je ein Drittel war.

Insgesamt können also innerhalb der Wählerschaft der Linkspartei Unterschiede festgestellt werden, jedoch überwiegen die Gemeinsamkeiten.

\section{Fazit: Kein baldiges Ende der Linkspartei}

Die meisten Befunde der Umfrage in den drei Städten deuten darauf hin, dass die Linkspartei hier bereits über ein relativ großes, dauerhaft mobilisierbares Wählerpotenzial verfügt. Inwieweit diese Aussagen über die Hochburgen in Oldenburg, Wilhelmshaven und Delmenhorst hinaus generalisiert werden können, steht damit noch nicht fest; jedoch spricht vieles für eine allgemeine Entwicklung, die zumindest auf andere größere Städte übertragen werden $\mathrm{kann}^{38}$.

Die aktuelle Diskussion um die Zunahme prekärer Beschäftigungsverhältnisse inmitten eines wirtschaftlichen Aufschwungs und die auseinandergehende Schere der Einkommensund Vermögensverteilung zwischen schlecht und gut Verdienenden ${ }^{39}$ trägt offenbar dazu bei, dass die Gerechtigkeitsfrage sich ein politisches Ventil sucht: in Form einer Stimmabgabe für die Linkspartei. Ihre zentralen Wahlkampfslogans zielten dementsprechend in genau diese Richtung ${ }^{40}$. Hinzu kommt, dass ein politisch aktives Segment der Wähler sich im Zuge der von vielen als ungerecht empfundenen Hartz III- und IV-Gesetzgebung vor allem von der SPD, aber auch von den Grünen abgewendet hat. Diese Bürger geben der Linkspartei auch an der Basis ein westdeutsches Gesicht, so dass diese auch für viele Westdeutsche wählbar wird.

Gegen eine kurzfristige affektive Protesthaltung der meisten Linksparteiwähler spricht vor allem, dass die Entscheidung zugunsten dieser Partei nicht auf einer einzigen Sachfrage beruht. Vielmehr scheint ein sozialstrukturell identifizierbares Bevölkerungssegment, das sich trotz seiner formal relativ hohen Bildungsabschlüsse ${ }^{41}$ selbst überwiegend den unteren

37 Ein Engagement der SPD für Mindestlöhne allein wird diese Wähler voraussichtlich nicht für die SPD zurückgewinnen.

38 In anderen städtischen Wahlkreisen Niedersachsens erreichte die Linkspartei Ergebnisse, die denen in den drei Städten sehr nahe kommen, zum Beispiel in Hannover-Linden 13,3, HannoverMitte 10,3, Braunschweig-West 10,4 und Göttingen-Stadt 10,4 Prozent.

39 Vgl. Die Gerechtigkeitslücke, in: Der Spiegel, Nr. 51 (2007).

40 Vgl. den Beitrag von RalfTils und Thomas Saretzki in diesem Heft der ZParl.

41 Der Befund, dass die Linkspartei in allen Bildungsschichten etwa gleich stark vertreten ist und lediglich unter Wählern mit formal höherer Bildung etwas überdurchschnittlich abschneidet, deckt 
Gesellschaftsschichten zuordnet, Präferenzen für die - jetzt auch in den westlichen Bundesländern als wählbare Alternative wahrgenommene - Partei zu entwickeln. Außerdem haben die Wähler der Linkspartei relativ konsistente Wertvorstellungen. Diese wirken politisch weitaus langfristiger als einzelne Sachfragen, die in der Tagespolitik diskutiert werden. Hinzu kommt die Unterstützung von vielen Wählern, die sich der Mittelschicht zuordnen und sich offenbar von sozialem Abstieg bedroht sehen. Damit spricht vieles dafür, dass ein neues Cleavage zwischen den Gewinnern und Verlierern der Modernisierungsprozesse der letzten Jahrzehnte entstanden ist ${ }^{42}$, das durch die Linkspartei repräsentiert wird. Somit werden sich Prognosen über ihr baldiges Ende ${ }^{43}$ mit hoher Wahrscheinlichkeit als falsch erweisen.

sich mit den Ergebnissen der Wahltagsbefragung der Forschungsgruppe Wahlen, vgl. Viola Neu, Landtagswahlen in Hessen und Niedersachsen am 27. Januar 2008, Online-Publikation der Konrad-Adenauer-Stiftung, http://www.kas.de/wf/doc/kas_12893-544-1-30.pdf (14. April 2008).

42 Hanspeter Kreisi, The transformation of cleavage politics, in: European Journal of Political Research, 33. Jg. (1998), S. $165-185$, S. 181 f.

43 Vgl. Viola Neu, Die Sonne geht im Westen auf: Die Fusion von WASG und PDS, Online-Publikation der Konrad-Adenauer-Stiftung, Berlin 2007, http://www.kas.de/wf/de/33.11146/ (3. März 2008).

\title{
Parlamentsvergrößerung als Problemlösung für Überhangmandate, Pattsituationen und Mehrheitsklauseln*
}

\author{
Friedrich Pukelsheim und Sebastian Maier
}

In Wahlsystemen, die eine Personenwahl mit einer Verhältniswahl verbinden, können Überhangmandate entstehen. Außerdem können Pattsituationen auftreten, und die Umsetzung der absoluten Stimmenmehrheit in die absolute Sitzmehrheit im Parlament ist nicht ohne weiteres gesichert. Zur Lösung dieser Probleme sollen im Folgenden Strategien zur Vergrößerung des Parlaments vorgestellt werden. Anlass für diese Vorschläge sind Initiativen im Landtag von Nordrhein-Westfalen, das Landeswahlgesetz zu novellieren. Statt der bisherigen, doppelt ausgewerteten Einzelstimme soll ein an das Bundeswahlgesetz angelehntes System mit zwei Stimmen eingeführt werden. Zudem soll für die Verrechnung von Stimmen in Sitze die Quotenmethode mit Ausgleich nach größten Resten (Hare / Nie-

\footnotetext{
Überarbeitung der schriftlichen Stellungnahme für die öffentliche Anhörung des Hauptausschusses des Landtags Nordrhein-Westfalen am 16. August 2007. Neben den Autoren, die Mathematiker sind, wurden der Politikwissenschaftler Uwe Andersen, die Verfassungsrechtler Thorsten Koch und Bodo Pieroth, der Geschäftsführer des Vereins Mehr Demokratie Daniel Schily und das Vorstandsmitglied der LandesschülerInnenvertretung Nordrhein-Westfalen Horst Wenzel angehört. Weitere Informationen sind unter www.uni-augsburg.de/pukelsheim/2007Duesseldorf bereitgestellt. Die Autoren danken Martin Fehndrich (www.wahlrecht.de) und Benjamin Beckmann für wertvolle Kritik und Anregungen.
} 